D.T. Wickramasinghe, L. Ferrario, and G.V. Bicknel!, eds.

\title{
Magnetically-Driven Winds from Protostellar Disks
}

\author{
Mark Wardle \\ Research Centre for Theoretical Astrophysics, University of Sydney
}

\begin{abstract}
Angular momentum transport in protostellar disks can be achieved by the action of a large scale magnetic field that runs vertically through the disk. The magnetic field centrifugally drives material from the disk surfaces into a wind, initiating a bipolar outflow. One apparent difficulty for this model is that the conductivity of the disk is extremely low in the inner $0.1-10 \mathrm{AU}$ of the disk, where grains are the dominant charge carriers. Near the midplane, charged grains are unable to drift through the neutral gas and there is negligible coupling between the magnetic field and the disk material.

However, the removal of angular momentum and acceleration of a wind by a magnetic field can still take place in the surface layers of the disk where the gas conductivity increases dramatically. Solutions to the multifluid MHD equations for the vertical structure of a disk at a particular radius are presented. Most of the disk material sits in hydrostatic equilibrium and does not interact with the magnetic field running vertically through it. Near the disk surfaces, the coupling between the magnetic field and disk material is sufficient to initiate an outflow from the disk surface.
\end{abstract}

\section{Introduction}

Disk-wind models for protostellar outflows are based on the self-similar solutions of Blandford and Payne (1982). Open magnetic field lines threading the disk vertically are carried around the central object, and material is centrifugally flung out along the field lines to form an outflow. The Blandford and Payne solutions and recent numerical simulations (Ustyugova et al. 1995; Meier, Payne and Lind 1996; Ouyed, Pudritz and Stone 1996), do not explicitly include the disk, instead assuming a steady injection of disk material at the base of the wind, and that the footpoints of the field lines are frozen into a Keplerian disk.

The microphysics determining the disk's conductivity is an integral part of the problem of accelerating outflows. Flux-freezing must break down within the disk, otherwise the magnetic field would be continuously advected towards the central object while being amplified by differential rotation. The finite conductivity of the disk is also intimately related to the loading of some of the material onto field lines at the disk surface and its injection into the wind (Wardle and Königl 1993). Perhaps most importantly, the disk conductivity places constraints on the ratio of the radial and azimuthal components of the magnetic field at the disk surface. For example, in the absence of radial field line drift the 
electric field within the disk is predominantly radial. The disk conductivity, a tensor, determines the ratio of the radial and toroidal current flowing within the disk in response to the electric field. In turn, the current determines the relative magnitude of the toroidal and radial field components at the disk surfaces. Thus the disk conductivity effectively imposes an additional boundary condition that has yet to be incorporated into the wind models.

\section{The conductivity and structure of protostellar disks}

Wardle and Königl (1993; herafter WK) examined the effect of the breakdown of flux-freezing in the outer regions of protostellar disks, beyond a few tens of AU from the central star. At these radii the dominant charged species are ions and electrons, and (as suggested by Königl 1989) the conductivity is low enough to permit significant ambipolar diffusion, but not so low that the coupling between the field and gas is negligible. WK constructed solutions for the vertical structure of the disk that could then be matched approximately onto self-similar wind solutions. Li (1996) has recently constructed analogous self-similar disk-wind solutions.

Several factors cause the conductivity to plummet within $10 \mathrm{AU}$ of the protostar as the gas density becomes successively higher. There are more neutral targets for drifting charged species to collide with; dust grains, which have a large neutral collision cross-section, become the dominant charged species (e.g. Nishi, Nakano and Umebayashi 1991); and the gas is self-shielding from cosmic rays once the surface density of the disk is larger than about $200 \mathrm{~g} \mathrm{~cm}^{-2}$, reducing the number of charged particles per unit volume. All of these effects reduce the current that can flow in the presence of an applied electric field. The conductivity partially recovers within about $0.1 \mathrm{AU}$ of the protostar where the disk temperature becomes sufficient to ionise potassium (Hayashi 1981; see also Li 1996).

Although the conductivity at the disk midplane is far too low to couple the field and disk material between 0.1 and $10 \mathrm{AU}$, cosmic rays can penetrate the disk to a depth of $100 \mathrm{~g} / \mathrm{cm}^{2}$, and there may be sufficient coupling between the field and the surface layers of the disk for magnetic effects to play an important role (Hayashi 1981; Gammie 1996). A plausible magnetic field structure is sketched in Figure 1. The magnetic field lines are straight in the "dead" region of very low conductivity, which cannot support a significant current. The surface layers, however, can support an accretion flow and accelerate an disk wind in much the same way as the original Wardle and Königl solutions.

New solutions have been obtained using the WK equations with some modifications: the charged species are dust grains rather than ions and electrons, the number density of charged particles scales as the square root of the gas density, rather than being constant, and the cosmic-ray ionisation rate decreases exponentially with depth into the disk, with a characteristic attenuation column of $96 \mathrm{~g} \mathrm{~cm}^{-2}$.

Figure 2 compares a typical WK solution (left panel) with a solution appropriate to conditions at $1 \mathrm{AU}$ (right panel). As expected, the new solutions show that the bulk of the disk material is inactive. Cosmic rays can maintain a reasonable ionisation fraction in the surface layers, where the solution resembles 


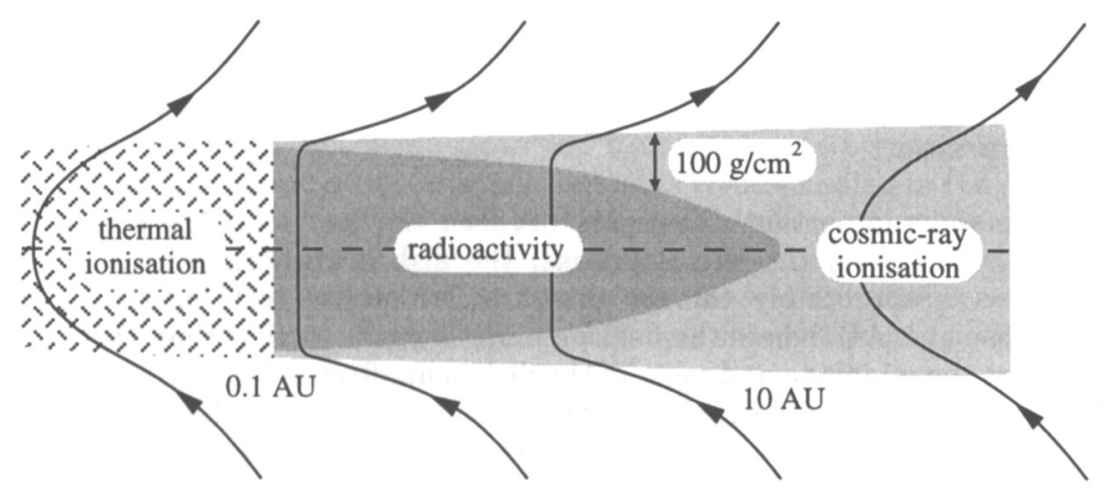

Figure 1. A sketch of the ionisation structure and magnetic field configuration expected for a protostellar disk. Cosmic rays can penetrate the first $100 \mathrm{~g} / \mathrm{cm}^{2}$ of the disk, so cannot maintain significant ionisation deep into the disk inside about $10 \mathrm{AU}$. Thermal ionisation of metals becomes significant within $0.1 \mathrm{AU}$ where the disk temperature exceeds $1000 \mathrm{~K}$. The magnetic field does not interact with the disk material in the region where radioactivity is the dominant form of ionisation because the conductivity there is negligible.
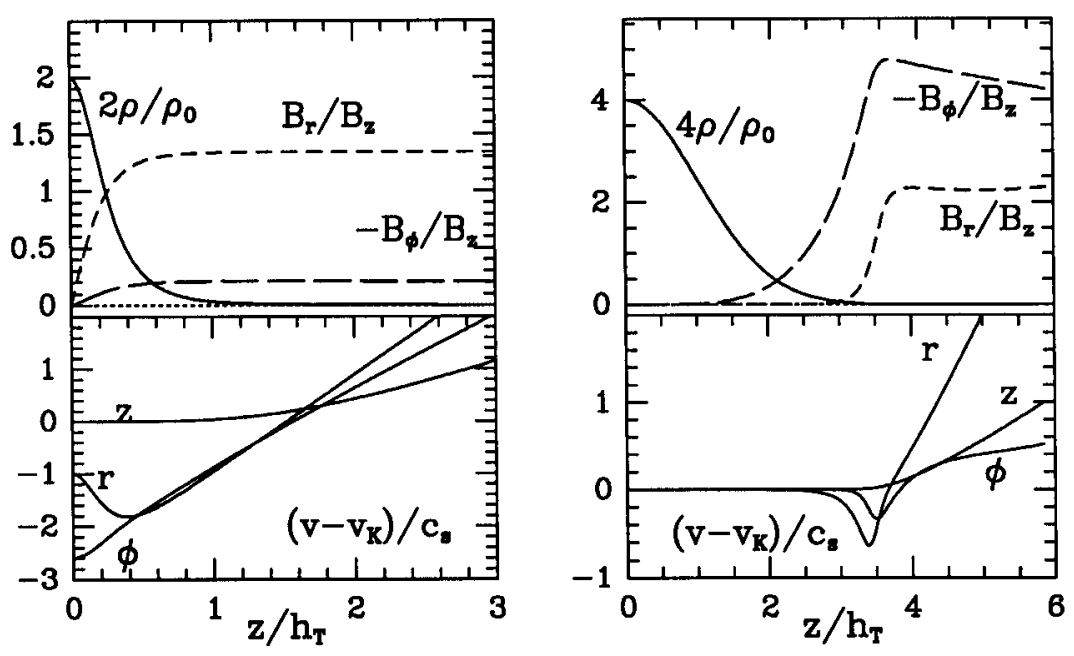

Figure 2. Vertical structure of a protostellar disk outside of $10 \mathrm{AU}$ (left) and at $1 \mathrm{AU}$ (right). The top panel of each figure plots the run of density and of the magnetic field components with height above the midplane in units of the isothermal scale height. The velocity components (relative to Keplerian and normalised by the sound speed) are plotted in the lower panels. 
the WK solutions; the magnetic field bending away from the rotation axis and centrifugally driving an outflow from the disk surface through a sonic point.

\section{Discussion}

There are some important differences between the two solutions presented in Figure 2. Firstly, in the WK solutions, the disk is compressed by magnetic stresses, so is appreciably thinner than the isothermal scale height. This is not the case at $1 \mathrm{AU}$, where the field pressure is much smaller than the thermal pressure at the midplane and most of the disk is unaffected by magnetic stresses. Secondly, Wardle and Königl showed that in the ambipolar diffusion dominated case, the toroidal field dominated the poloidal field at the disk surface. In the present case, the opposite is true.

The solution for the vertical structure at $1 \mathrm{AU}$ is not strictly correct as the gas density at the sonic point is of order $10^{10} \mathrm{~cm}^{-3}$, comparable to the midplane density at $30 \mathrm{AU}$, and ions and electrons are again important. Work towards solutions incorporating multiple charged species (i.e. ions, electrons, and charged grains) is underway.

Acknowledgments. This work forms part of a collaborative effort with Arieh Königl.

\section{References}

Blandford, R. D., \& Payne, D. G. 1982, MNRAS, 199, 883

Gammie, C. F., 1996, ApJ, in press

Hayashi, C. 1981, Prog. Theor. Phys. Suppl. 70, 35

Königl, A. 1989, ApJ, 342, 208

Li, Z.-Y. 1996, ApJ, 465, 855

Meier, D. L., Payne, D. G., \& Lind, K. R. 1996, in Extragalactic Radio Sources, in press

Nishi, R., Nakano, T., \& Umebayashi, T. 1991, ApJ, 368, 181

Ouyed, R., Pudritz, R. E., \& Stone, J. M. 1996, Nature, submitted

Ustyugova, G. V., Koldoba, A. V., Romanova, M. M., Chechetkin, V. M., \& Lovelace, R. V. E. 1995, ApJ, 439, L39

Wardle, M., \& Königl, A. 1993, ApJ, 410, 218

\section{Discussion}

Th. Henning: You talked about dust grains and relative velocities. Would you not expect strong interactions between the grains leading to the growth of the particles? This should strongly influence your picture.

$M$. Wardle: The relative velocities of charged grains are small and the fraction of charged grains is also small, so the drifts in the model do not affect the grain evolution. The evolution of grains - settling, coagulation, growth of ice mantles 
will affect the disk conductivity however. The model does not invoke turbulence, and the lack of turbulence affects the time scales for these processes.

D. Meier: I am very pleased to see work being done on the interior magnetic structure of the accretion disk. However, I am not certain how relevant such work may be to the production of winds and jets. We are finding in the numerical models that, no matter what the initial field configuration assumed, an outflow still results. The reason is that above the disk the field re-adjusts itself, usually by being dragged by various radial flows, into the configuration necessary to drive a wind or jet. We see this even in the simple numerical models $(2 \mathrm{D}$ "wind only" calculations).

M. Wardle: The behaviour seen in the simple numerical models is a result of assuming that the field lines are frozen into a Keplerian disk, and that the disk injects mass into the wind at a constant rate. There is no back-reaction on the disk in the form, of diffusion of field lines, through the disk or a shutting off of the mass injection into the base of the wind. Under these circumstances the field just above the disk can always be wound up and force an outflow.

The disk and wind numerical models suffer from low dynamic range in density and the assumption of ideal MHD (apart from numerical resistivity).

Both types of numerical models are instructive but the disk is an integral part of the problem. 